\title{
Evolution of dwarf-elliptical galaxies
}

\author{
G. Hensler ${ }^{1,2}$, Ch. Theis ${ }^{1,2}$, and J. S. Gallagher, III. ${ }^{3}$ \\ 1 Institut für Theoretische Physik und Astrophysik, Universität Kiel, Olshausenstr. 40, 24098 Kiel, Germany \\ 2 New address: Institut für Astronomie, Universitäts-Sternwarte Wien, Türkenschanzstr. 17, 1180 Vienna, Austria \\ e-mail: hensler@astro.univie.ac.at \\ 3 Department of Astronomy, University of Wisconsin-Madison, 5534 Sterling Hall, 475 N. Charter St., \\ Madison, WI 53706-1582, USA
}

Received 14 March 2000 / Accepted 4 May 2004

\begin{abstract}
Here we present as an extension to already published models of massive spherical galaxies one-dimensional chemodynamical models of dwarf elliptical galaxies in the mass range between $10^{9}$ and $10^{10} M_{\odot}$ and initial density fluctuation of $1 \sigma$ and $3 \sigma$. Because of their vanishing angular momentum the models are restricted to spherical symmetry. Due to this limitation the dynamics of the different components consider only radial motions and also their interaction processes have only one degree of freedom. Therefore, the galaxy evolution is preferably determined by radial oscillatory phenomena caused by heating and cooling of the interstellar gas, which reenforce effects like starbursts. Nevertheless, the low gravitational binding energy of dwarf ellipticals can easily be exceeded by thermal and turbulent energy production in the interstellar medium, leading to gas expansion and even to galactic winds. Furthermore, gas phases as well as gas and stars can decouple dynamically during the galactic evolution. For comparison with observational signatures like stellar kinematics, radial densities and metallicity distributions of the different components, the chemo-dynamical treatment of galaxy evolution must take the multi-phase character of the interstellar medium and self-regulation of star formation at low gravitation into account.

Since non-rotating low-mass galaxies with stochastic star-formation episodes are seen as dwarf ellipticals, the aim of this paper is to compare the $1 \mathrm{~d}$ chemo-dynamical models with observed characteristics of this type of dwarf galaxies. Several features like stellar populations from separate formation episodes, low metallicities, significant mass loss, etc. can be reproduced by the models in a global manner. Quantitative disagreements between model predictions and observations provide insight into necessary improvements of subsequent chemo-dynamical models implying rotation, dark matter halos, and external effects by an intergalactic gas, like its pressure, gas infall and stripping of galactic gas. The results can be summarized as follows: $10^{9} M_{\odot}$ models are characterised by a single star-formation event during the initial collapse with an active self-regulation leading to reexpansion and massive gas loss as well as remaining at very low metallicities; $10^{10} M_{\odot}$ dwarf elliptical models become almost gas-free due to large initial star formation and a strong galactic wind. These as well as a $5 \times 10^{9} M_{\odot}$ and $3 \sigma$ model experience oscillatory but non-negligible later star-formation histories that can account for the observed intermediate-age stellar populations.
\end{abstract}

Key words. galaxies: dwarf - galaxies: elliptical and lenticular, cD - galaxies: evolution - galaxies: general

\section{Introduction}

Dwarf elliptical galaxies (dEs) are the most common and astrophysically interesting class of galaxies in the local universe (see Ferguson \& Binggeli 1994, for review). They are frequently cited as examples of "stellar fossil" systems in which the bulk of their star formation occurred in the past; these characteristics led Baade (1944) to use Local Group dEs as the archetypes of Population II systems. Most known dEs are located in regions with high galaxy densities, and dEs are the most numerous type of galaxy in nearby galaxy clusters, including Coma (Thompson \& Gregory 1993; Secker et al. 1997), Fornax (Caldwell 1987; Rakos et al. 2001), Virgo (e.g., Binggeli et al. 1985), and recently Perseus galaxy clusters (Conselice et al. 2003a). Most cluster dE galaxies are probably free of interstellar gas and contain few young stars (Bothun et al. 1985).

In trying to understand the $\mathrm{dE}$ galaxies, we must also consider several regularities in their structures. A positive correlation also exists in dEs since years between optical surface brightness and luminosity (e.g., Binggeli et al. 1984; Wirth \& Gallagher 1984; Kormendy 1985) and between luminosity and stellar velocity dispersion. The latter parameter also correlates with metallicity (e.g., Dekel \& Silk 1986, hereafter: DS86; Petersen \& Caldwell 1993). Furthermore dE galaxies often have flattened profiles in combination with a low ratio of stellar velocity dispersion to rotation speed implying that their shapes reflect an anisotropic velocity dispersion rather than rotation (Bender \& Nieto 1990; Bender et al. 1991). 
The dEs therefore are good choices for initial simulations with one-dimensional galaxy models.

The combination of low gas mass fractions and moderateto-low stellar metallicities in $\mathrm{dE}$ and the related dwarf spheroidal (dSph) galaxies is a key feature of this class (Grebel et al. 2003). In galaxies where gas is depleted by star formation stellar abundances are predicted to be near the solar value (Tinsley 1973; Pagel 1997). The lower abundances seen in stars in dEs (about 0.1 of solar or less; Gallagher \& Wyse 1994; Cellone \& Forte 1996; Han et al. 1997) suggest that extensive gas loss occurred during the evolution of dEs and star formation ceased due to a lack of raw materials rather than exhaustion of the gas supply through star formation. Galactic winds are therefore a hallmark of modern models for $\mathrm{dE}$ galaxies, beginning with the studies by DS86 and Vader (1986), and they are commonly assumed to have cleaned out $\mathrm{dE}$ galaxies soon after their formation.

A variety of observations are now available which bring this standard picture for the evolution of $\mathrm{dE}$ galaxies into question. Recent HI studies of Virgo cluster dEs (see e.g. Conselice et al. 2003b) have unveiled that a small but significant fraction of them contains gas, has experienced recent star formation, and can be argued from internal kinematics and cluster distribution data to form an infalling class of different types of gas-rich galaxies in the state of morphological transformation. In addition, ultra-compact DGs have been detected and classified as a new type of cluster dEs that differ by their intrinsic structure and brightness (Phillipps et al. 2001). The situation in Local Group dEs is similarly complicated, with NGC 185 and NGC 205 both being well known to have cool interstellar gas and trace young stellar populations, while NGC 147 contains neither an ISM nor young stars (Hodge 1971; Young \& Lo 1997). Furthermore, high-quality color-magnitude diagrams for nearby $\mathrm{dSph}$ and $\mathrm{dE}$ galaxies often reveal substantial intermediate age stellar populations (Smecker-Hane 1997; Han et al. 1997; Martínez-Delgado \& Aparicio 1998; Hurley-Keller et al. 1998; Stetson et al. 1998; Ikuta \& Arimoto 2002) which means that small spheroidal galaxies did not form all their stars in single events near the time of giant galaxy formation. This agrees with the findings of large internal metallicity dispersions in local dSphs from high-resolution stellar spectra (Shetrone et al. 2001; Tolstoy et al. 2003). A second and possibly related line of evidence has come from deep surveys of faint galaxies. The frequent presence of compact, narrow emission line galaxies in moderate redshift galaxy samples (Koo et al. 1995; Guzman et al. 1996) leads naturally to the suggestion that such galaxies are small systems experiencing major starburst events perhaps 5-8 Gyr before the present, and likely predecessors of the current dE systems (Babul \& Rees 1992; Babul \& Ferguson 1996).

These new results provide the motivation for a reexamination of theoretical models for the evolution of small spheroidal galaxies. In this paper we discuss results from the simplest class of 1-dimensional chemo-dynamical models for the evolution of spheroidal dwarfs. An initial discussion of these results was presented by Hensler et al. (1993) but at that time much of the modern data on $\mathrm{dE}$ star formation histories had not yet been obtained. The basic precepts of chemo-dynamical models for galaxy evolution are reviewed by Hensler (2003) (see also Samland et al. 1997, hereafter: SHT). In this approach stellar metallicity levels provide tracers of stellar nuclear power inputs which in turn influence the structure of a multi-phase interstellar medium (ISM). These models thus build from the approach to galaxy evolution pioneered by Larson (1974) in directly dealing with the complex but inevitable interplay between gas and stars within evolving galaxies. Numerical models for spheroidal dwarf galaxies with some similar characteristics to those presented here have been calculated by Spaans \& Norman (1997); Mori et al. (1999); Lia et al. (2000); Carraro et al. (2001).

In Sect. 2 we describe the 1d chemodynamical models of spheroidal dwarf galaxies without dark matter. Models are now in progress to include dark matter and with two dimensions (with the prescription of SHT) as well as to proceed to a more detailed chemical abundance treatment (e.g., Hensler \& Rieschick 1999) and to three dimensions (Berczik et al. 2003; Harfst et al. 2003). Section 3 presents results of the model calculations, and Sect. 4 compares the observable properties of models. Conclusions are given in Sect. 5.

\section{Chemo-dynamical treatment}

Our models are based on the chemo-dynamical prescription of galaxy evolution (Hensler 1987; Theis et al. 1992, hereafter: TBH; Hensler 2003). This approach involves following the energy and metal flows from stars into a multi-phase representation of the galactic ISM. Chemo-dynamical models include effects of the galactic gravitational potential, as well as stargas interactions via star formation, stellar radiation, and stellar mass loss which can dominate energetically the gravitation over part of the evolution. The state of the ISM and the mixing of elements in turn depends on phase transitions between a cool cloudy medium (CM) and a hot, diffuse intercloud medium (ICM) with $T \geq 10^{4} \mathrm{~K}$. Since several key processes like plasma cooling and stellar wind mass-loss rates depend on metallicity, but the metal injection occurs diversely to the different gas phases depending on the energetical process of its release, the stellar population can store evolutionary information through the stellar metallicity-age relationship and the time dependent abundance ratios.

Our models inherently include the chemical abundances as functions of time and galactic radius for stars, the ICM, and the CM. Detailed descriptions of this technique as applied to one-dimensional models such as those discussed here are given in TBH. While the TBH paper has concentrated on massive galaxies of $10^{11} M_{\odot}$ and above and addressed the question as to how and in what manner the collapse of giant ellipticals (gEs) could be reproduced by the dissipational $1 \mathrm{~d}$ collapse of a gaseous protogalaxy, here we wish to extend this $1 \mathrm{~d}$ chemo-dynamical model to low-mass galaxies which should be well-represented by such models. 1d hydrodynamical models by Friaca \& Terlevich (1998) that studied also the collapse of massive ellipticals are in good agreement with ours (TBH) and could be fairly compared with observations of gEs and QSOs. Since they treat only a single gas phase, this shows that massive gravitational potentials dominate the energetics over most of 
the evolution. This relation changes presumably for low-mass galaxies where the binding energy is easily exceeded by the stellar energy release.

Because our models begin as purely gaseous protogalaxies, it might be expected that the results depend on the functional representation of the stellar birth law. The dE models assume a Schmidt-type stellar birth law, $\Psi=C_{n} \rho_{\mathrm{CM}}^{n}$, where the $\mathrm{CM}$ density is $\rho_{\mathrm{CM}}$. However, since $\rho_{\mathrm{CM}}$ depends in part on the recent history of star formation, the model star-formation rates (SFRs) often are self-regulated and thus are insensitive to the detailed functional form of $\Psi$ (see TBH; and Köppen et al. 1995). For the models presented here we chose $n=2$ over a stellar mass range of $0.1-100 M_{\odot}$, for which the mass distribution is assumed to be the standard Salpeter initial mass function.

A summary of the prescription for stellar energetics adopted here is also given by TBH. We note that stars with initial masses of $\geq 10 M_{\odot}$ become type II supernovae (SNeII) at the end of their lives while type Ia supernovae are assumed to stem from binary stars at a rate normalized to observed SNIa rates (Tammann 1982). We adopt an energy injection of $10^{51}$ erg per SN and follow the TBH prescription for metal yields. Köppen et al. (1998) have also proven that the evaporation/condensation competition adjusts at self-regulated star formation equilibrium because of the feedback of hot-gas production by means of SNeII to the SFR. Most of the models presented here were computed with a drag coefficient of 0.01 . This parameter determines the transfer of momentum between flows of the hot ICM and the CM.

The family of $1 \mathrm{~d}$ chemo-dynamical models of low-mass galaxies is most suitable for representations of non-rotating, symmetrical systems, such as dEs. Furthermore, in low mass galaxies which originate from $3 \sigma$ or lower amplitude density fluctuations in a cold dark matter cosmology, the gravitational potential is too flat and too shallow to contain the effects of SN blasts within the galaxy (DS86). Under these conditions a galactic wind may occur, thereby producing substantial mass loss (MacLow \& Ferrara 1999) and subsequent re-expansion of the model galaxy (Burkert \& Hensler 1989). Even if dark matter (DM) is present in real galaxies, a re-expansion of the stellar component will occur as long as the central DM density is much lower than that of the gas. Thus our model may not apply in some dSphs where cores have high DM densities (see Gallagher \& Wyse 1994).

\section{Models for diffuse dwarf E galaxies}

In present cosmological scenarios small galaxies ought to formed as the oldest objects if cold dark matter (CDM) fluctuations harbor sufficient baryonic substrate. Later on larger galaxies are assembled by hierarchical accumulation of smaller units. Since the gas must cool and, subsequently, collapse so that those condensations led to enhanced star formation such scenarios would expect a very early epoch of DG formation and their early appearance in the universe. This, however, stands in conflict with observations which show that the bulk of stars in DGs have intermediate ages (see reviews by Mateo 1998; Grebel 1999), and thus seem to have formed at around $z=1$.
Even the dSphs with purely old stellar populations show evidence for star formation extendng over several Gyr (e.g., Ikuta \& Arimoto 2002; Grebel et al. 2003). This obvious inconsistency is not yet resolved, but could be due to the impact of the intergalactic UV radiation field that slowed the evolution of DG formation (e.g., Thoul \& Weinberg 1996; Kepner et al. 1997; Barkana \& Loeb 1999). Since our aim here is to consider isolated entities as a first chemo-dynamical approach to DG evolution, i.e. systems unperturbed by external effects and without inherently implied absolute timescales, on the one hand, our model evolution can be considered to start after the delayed recombination of the embedded gas. Moreover, the existence of a dark matter (DM) halo is expected to accelerate the collapse and to lead to enhanced star formation (Mori et al. 1999). Since the initial conditions are uncertain and because this first strong gravitational collapse caused by gas cooling should be automatically self-regulated by the star formation itself due to local energy release of the first generation of stars at least by the cumulative explosions of SNeII, we aimed to follow the globally simplest, but locally well detailed treatment of galaxy evolution. Particular results that are expected to be affected by either the background radiation field or a DM halo will be discussed at the end.

Also Mori et al. (1999) investigated the evolutionary differences between dwarf and giant ellipticals in a CDM universe applying a single-gas phase SPH code to density fluctuations of total masses of $10^{10}$ and $10^{12} M_{\odot}$ where $10 \%$ consists of baryonic matter. Although their models have been followed for $10^{9}$ yrs only, the results of their low-mass model will be compared with our corresponding model.

\subsection{Initial conditions}

The initial conditions are chosen analogously to the $1 \mathrm{~d}$ chemodynamical models of the more massive galaxies published in TBH: the models start with a purely gaseous, isotropic, and homogeneous non-rotating sphere in virial equilibrium. The homogeneity concerns only the radial mass density which, on the other hand, inherently contains the gaseous inhomogeneity by the division into a diffuse hot ICM and the embedded cooler clouds (CM). A DM halo is neglected here for reasons of consistency with the massive models (TBH).

The initial temperatures of the gas phases are set to $3 \times$ $10^{4} \mathrm{~K}(\mathrm{ICM})$ and $8000 \mathrm{~K}(\mathrm{CM})$, respectively, with a CM volume filling factor of $20 \%$. The initial metallicity is set to a low, but arbitrary value of $5 \times 10^{-4} Z_{\odot}$. Reasonably, two parameters must be specified: the initial gas density $\rho$ and the initial gas mass $M$. As extreme cases we consider two values of initial densities derived from cosmological density fluctuations (DS86): low-density fluctuations of $1 \sigma$ and high-density fluctuations of $3 \sigma$.

For a better understanding and providing an insight to what extent the evolutionary behaviour depends on the initial conditions alone, we have structured the paper in the following manner: starting from a low-mass model of $10^{9} M_{\odot}$, where we discuss the differences between the initial densities only marginally because of almost the same global evolutionary 


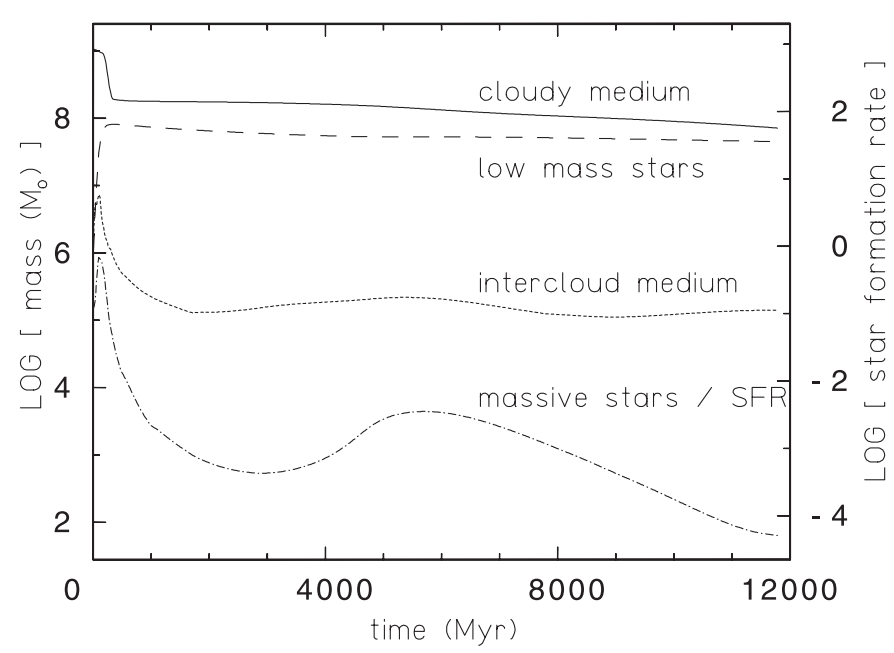

Fig. 1. The temporal evolution of mass distributions (left scale) in a $10^{9} M_{\odot}$ spherically symmetric galaxy originating from a $1 \sigma$ density fluctuation. While low-mass stars (dashed line), cloudy interstellar medium (thick full line) and intercloud medium (thin dotted line) are drawn individually, the massive star line (dash-dotted) represents simultaneously the star-formation rate (right scale; given in $M_{\odot} \mathrm{yr}^{-1}$ ). Reduction of the total mass is discernible from the cloudy medium.

tendency, we proceed to a more detailed presentation of $5 \times$ $10^{9} M_{\odot}$ models where the influence of the initial density becomes apparent, and finish with $10^{10} M_{\odot}$ models.

\section{2. $10^{9} M_{\odot}$ model}

For this model of lowest mass in this presentation we consider the evolution of both $1 \sigma$ and $3 \sigma$ initial perturbations. In its initial state the model consists entirely of gas which is cooling and collapsing under self-gravity. These low-mass systems thus produce an initial starburst followed by a declining SFR. This initial triumph of activity leads to a peak SFR of approximately $0.6 M_{\odot} \mathrm{yr}^{-1}$ over about $200 \mathrm{Myr}$ to produce $<10^{8} M_{\odot}$ of stars (Fig. 1). Due to the low gravitational energy, mass loss is the dominant feature of these models, so that only $10 \%$ of the initial mass becomes stars in the $3 \sigma$ model and only $8 \%$ in the $1 \sigma$ model. After the expulsion of most of the gas only a little falls back and produces as a characteristic feature of these low-mass models a second much weaker star-formation episode concentrated to the central region (see Fig. 1). Although the CM remains the major mass fraction (Fig. 1), its radial distribution (Fig. 2) demonstrates that in the innermost galaxy region its mass fraction amounts to $10 \%$ only so that one can say that they are gas-poor systems but not devoid of gas like real dEs or dSphs. The gas sitting at large radii can, on the other hand, already be interpreted not to belong to the $\mathrm{dE}$ anymore.

Since the global behaviour is the same for both density fluctuations, only the properties of the $1 \sigma$ model are shown here in Figs. 1-2. For the $3 \sigma$ model only the star-formation timescale and thus the evolutionary timescale are shortened by almost a factor of two.

The radial distribution of star formation in the low-mass galaxies is not very centrally concentrated; these galaxies would never look like blue compact dwarfs.

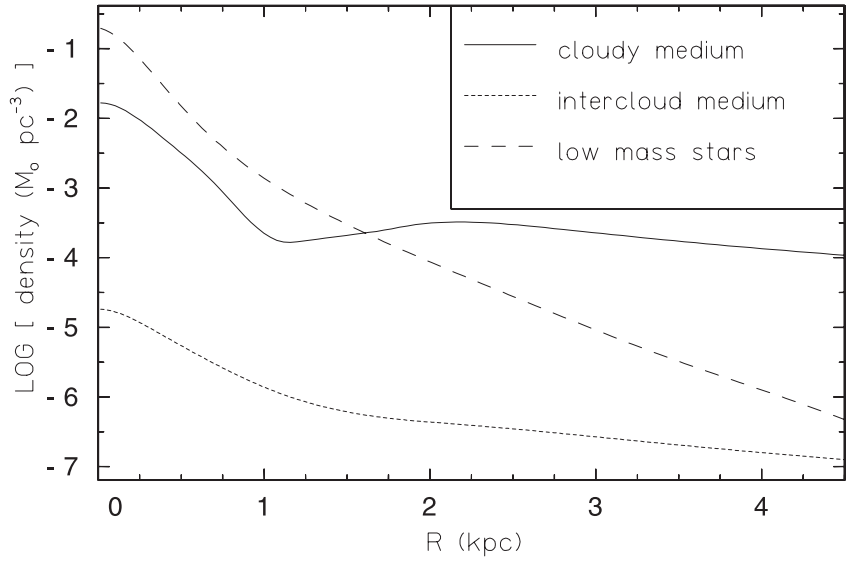

Fig. 2. Radial density distribution of cloudy and intercloud medium as well as low-mass stars after an age of $10 \mathrm{Gyr}$ of the same $10^{9} M_{\odot}$ model as in Fig. 1.

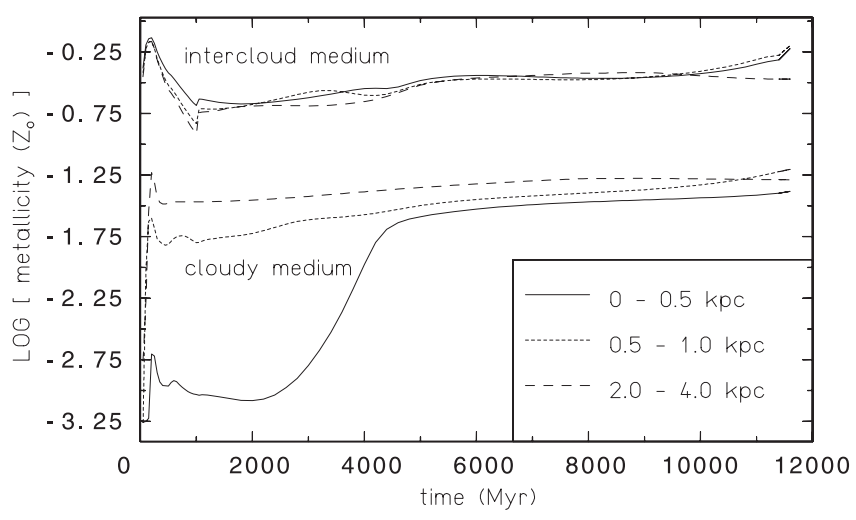

Fig. 3. Temporal evolution of the metallicity of cloudy (lower curves) and intercloud medium (upper lines) at different radial bins of the same $10^{9} M_{\odot}$ model as in Fig. 1.

The radial metallicity distribution can be most interestingly demonstrated for the $1 \sigma$ model of $10^{9} M_{\odot}$ (Fig. 3): the metal enrichment is predominantly stored in the ICM and enhances the $\mathrm{CM}$ abundances with a time delay due to phase transitions. As discernible in Fig. 3 the CM metal content is at all times higher at larger radii. This discrepancy between e.g. the central half kpc and the outermost region is largest during the first 4 Gyr i.e. during the most active star-formation phase. This is accomplished because the metals released by supernovae type II in the innermost region can only be partly deposited there. The supernova gas, namely, is too hot to condense, instead it evaporates parts of the $\mathrm{CM}$ and expands. By this, metals produced by different progenitor stars are well mixed and carried to larger radii until the outflowing ICM cools sufficiently, condenses onto clouds and pollutes the CM with inherited metals. Such a behaviour is a characteristic issue of the chemodynamical treatment. In galactic disks (SHT97) the same is also achieved during the early evolutionary stages and accounts for the $\mathrm{G}$ dwarf problem. Simple chemical models without any consideration of the gasdynamics and where the SFR depends on the gas density would, in contrast, always produce negative metallicity gradients. 


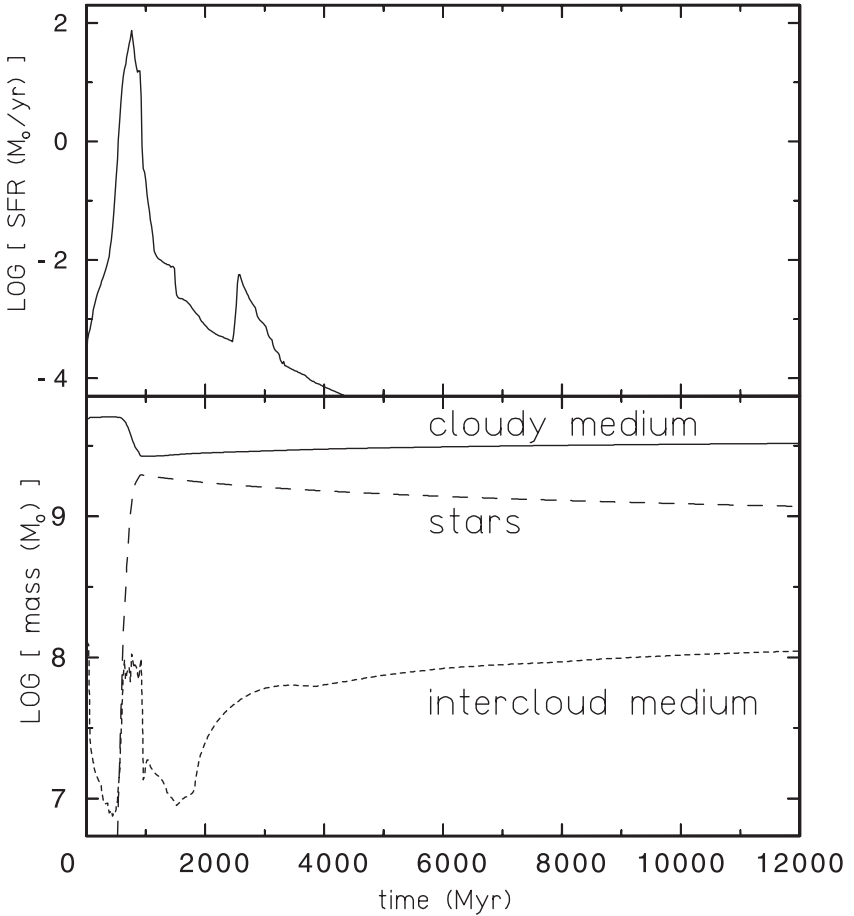

Fig. 4. Star-formation rate (upper panel) and masses (lower panel) of the $5 \times 10^{9} M_{\odot}$ model from a $1 \sigma$ fluctuation. Remarkably the gas fraction remains dominant although it cannot produce further stars. $40 \%$ of the mass is expelled by a galactic wind.

\section{3. $5 \times 10^{9} M_{\odot}$ model}

As an intermediate-mass $\mathrm{dE}$ we follow the evolution of a $5 \times 10^{9} M_{\odot}$ model from a $1 \sigma$ and a $3 \sigma$-density fluctuation, respectively. A comparison of the SFRs shows at a first glance that the star formation of the lower density model (Fig. 4) starts more slowly and extends to more than one billion years while the $3 \sigma$ model collapses much more rapidly and ignites its star formation within the first $300 \mathrm{Myr}$ (Fig. 5). Surprisingly, both peak SFRs reach almost $100 M_{\odot} \mathrm{yr}^{-1}$. After this first starburst the SFR decreases to insignificance for the $1 \sigma$ model and even ceases after about $3 \mathrm{Gyr}$, because $40 \%$ of the gas are expelled after this initial burst (see lower part of Fig. 4) and the remaining gas is too dilute to cool and contract. In contrast, the $3 \sigma$ model exerts a higher gravitational binding so that with only $20 \%$ mass loss the SFR drops after the same initial burst not to zero, but remains on a significant value of almost $1 M_{\odot} \mathrm{yr}^{-1}$ for 1 billion years and enters an oscillatory stage for another Gyr with an amplitude of about one order of magnitude (Fig. 5).

In Fig. 6 also the radial concentration of the star-forming region is visible over the evolutionary sequence by four snapshots at different times. The SFR shows a radial decline already after 100 Myr. During the oscillatory star-formation phase the star-forming regime is still extended and shrinks strongly when the total SFR drops drastically after 4 Gyr because of gas exhaustion. As in all the models (and revealed by the radial metallicity distribution of the $10^{9} \mathrm{M}_{\odot}$ model) the metal enrichment is predominantly stored in the ICM and enhances the CM abundances with a time delay due to phase transitions. Due to the inclusion of phase transition between ICM and CM as in the

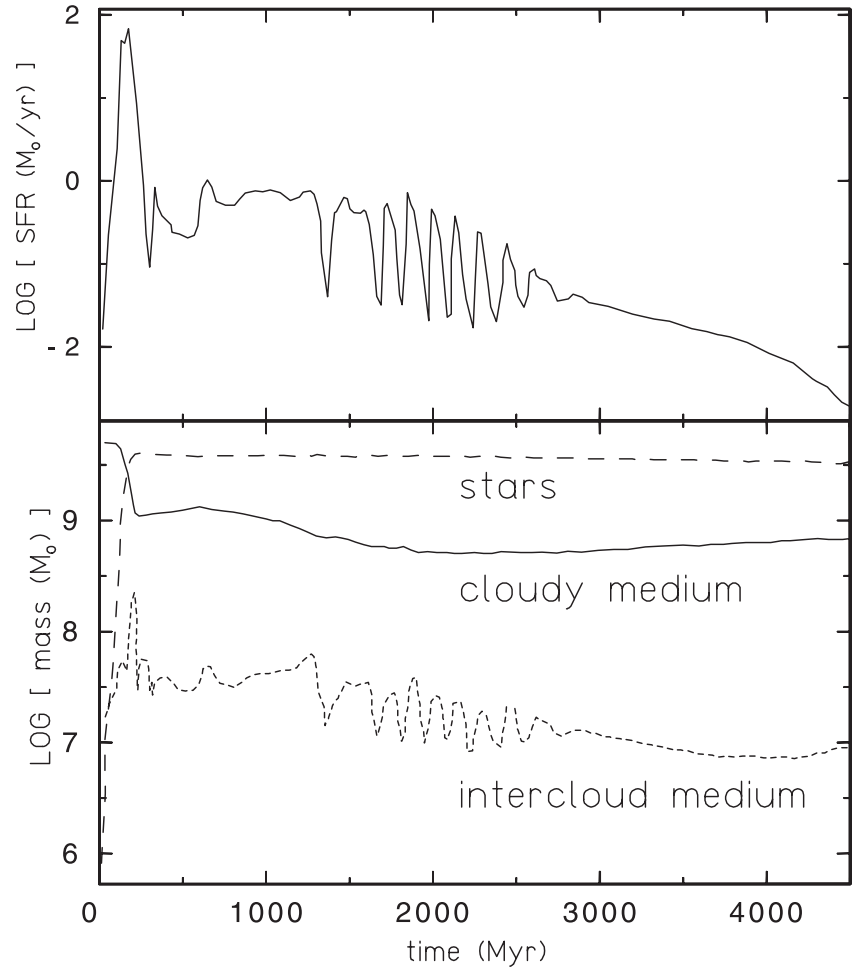

Fig. 5. Star-formation rate (upper panel) and masses (lower panel) of the $5 \times 10^{9} M_{\odot}$ model from a $3 \sigma$ fluctuation. See text for discussion. For comparison with Fig. 4 notice the different time axes!

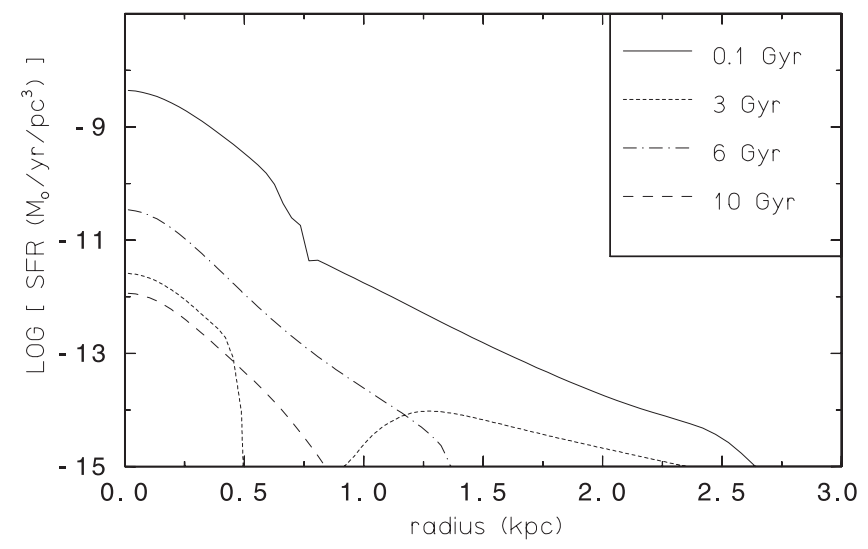

Fig. 6. Radial distribution of the star-formation rate at different times during the evolution of the same $5 \times 10^{9} M_{\odot}$ model as in Fig. 5 . The radial concentration of the star-forming region is discernible.

$10^{9} M_{\odot}$ model, the $\mathrm{CM}$ is reduced in the central region by means of evaporation, because of the hot state of the ICM due to intense SN production during the SFR peak events. This reduces the SFR according to the self-regulation picture (Köppen et al. 1998). While hot ICM is streaming out, by this, relaxing the evaporation situation, clouds can replenish the central region and further enhance the star formation. Consequently, also outward propagating shells of reduced SFR are achieved (see curve in Fig. 6 at $3 \mathrm{Gyr}$ ). Not before the ICM cools at larger radii condensation of metal-enriched ICM pollutes the clouds, leading to a radial metallicity increase because of the radially decreasing CM mass. 


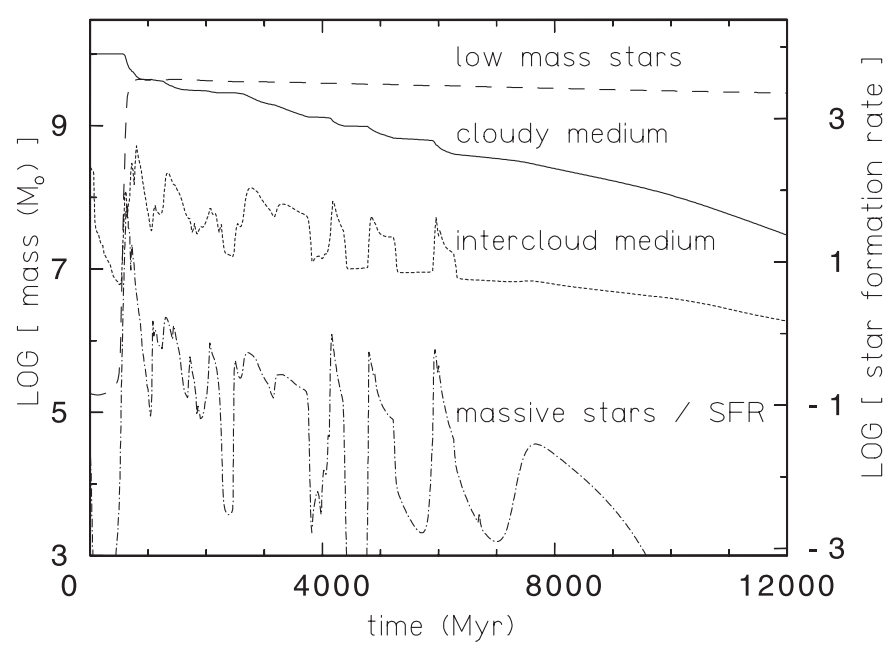

Fig. 7. The temporal evolution of mass distributions (left scale) in a $10^{10} M_{\odot}$ spherically symmetric galaxy originating from a $1 \sigma$ density fluctuation. While low-mass stars (dashed line), cloudy interstellar medium (thick full line) and intercloud medium (thin dotted line) are drawn individually, the massive star line (dash-dotted) represents simultaneously the star-formation rate (right scale in $M_{\odot} \mathrm{yr}^{-1}$ ). Reduction of the total mass due to a galactic wind is discernible from the cloudy medium. It coincides temporarily with the peak starformation rate.

Both features, central hole of stellar metal enhancement and outward propagating shells, respectively, are also visible in the low-mass models by Mori et al. (1999), although their SPH treatment accounts for one gas phase only. This qualitative agreement of different descriptions demontrates that this early evolutionary phase is dominated by the hot gas in the central few hundred parsecs. While the stellar metal enhancement at large radii in the chemo-dynamical models is caused by condensation and mixing of hot gas with the star-forming CM and therefore is self-consistently regulated by the state of the gas phases, in the Mori et al. (1999) model the higher abundances reflect directly the total metallicity of the gas. There the stars are formed in outward propagating shocks.

One can summarize the main features of $5 \times 10^{9} M_{\odot}$ chemodynamical models as follows: the lower density $1 \sigma$ fluctuation model ceases to form stars after an initial peak. This behaviour is a reflection of the lower gas density where selfregulation occurs so that more gas remains available than in the denser model. The $3 \sigma$ model consumes most of its gas in an initial rapid starburst, but some gas remains gravitationally bound and supports oscillating SFRs with time scales of about 300 Myr. These details of the star formation history would be difficult to observe in present-day DGs.

\section{4. $10^{10} M_{\odot}$ model}

As a last representative model we take a $1 \sigma$ initial density fluctuation for $10^{10} M_{\odot}$ according to DS86 and then follow the evolution of this isolated object for more than $10 \mathrm{Gyr}$. The evolution of the model is summarized in Figs. 7 and 8.

Evolution begins with an initial collapse and the onset of low-level star formation. This early quiescent phase lasts for

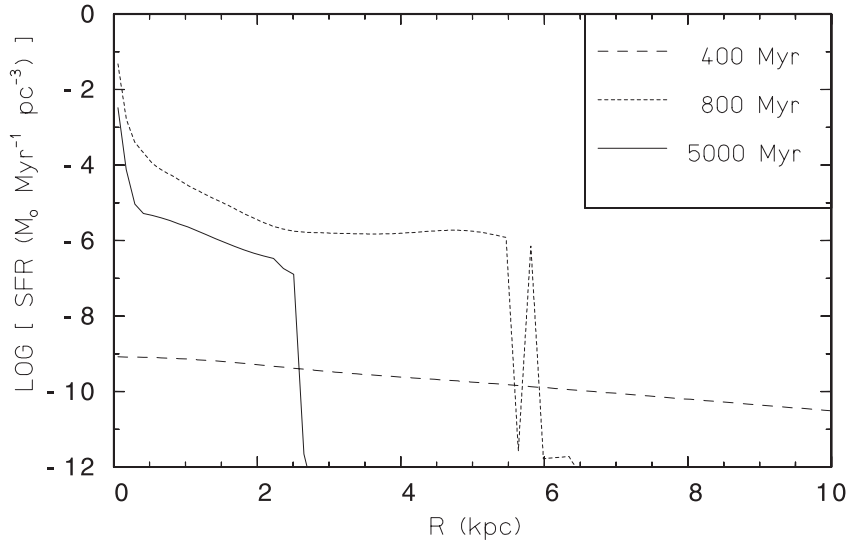

Fig. 8. Radial distribution of the star-formation rate at different times during the evolution of the same $10^{10} M_{\odot}$ model as in Fig. 7. The radial concentration of the star-forming region is perceivible.

slightly longer than one free-fall time for the proto-galaxy of $t_{\mathrm{ff}}=383$ Myr. Soon thereafter a violent initial starburst occurs. A peak $S F R$ of about $100 M_{\odot} \mathrm{yr}^{-1}$ is sustained for about $60 \mathrm{Myr}$, and the $S F R$ declines below $10 M_{\odot} \mathrm{yr}^{-1} 200 \mathrm{Myr}$ after the beginning of the starburst. This active phase is followed by a 3 Gyr plateau during which the SFR oscillates between 0.01 and $1 M_{\odot} \mathrm{yr}^{-1}$ with a rough period of a dynamical timescale. At the end of the SFR plateau phase at an age of almost 6 Gyr, the bulk of the stars have been formed and the system is seriously depleted in CM gas.

The late star-forming evolution is characterized by a lowpeak intensity $\left(\leq 0.1 M_{\odot} \mathrm{yr}^{-1}\right)$ and high-amplitude variations in the SFR. These events occur because the decreased density of the ISM leads to an increase in cooling time to more than a dynamical timescale so that the collapse halts and even reverses. Therefore, not before the gas has cooled again can the infall proceed and the SFR rise again. At high SFR strong gas flows between CM and ICM are induced. After about 9 Gyr the amount of ISM within the galaxy is reduced to the point where smoothly declining star-forming activity occurs only at very low levels and becomes totally insignificant after 10 Gyr. Thus a key feature of these models is that the bulk of the stars in $d E$ galaxies should have ages of $\geq 8$ Gyr for galaxies which formed 10 Gyr ago. This behaviour becomes even more extreme for the $10^{10} M_{\odot} 3 \sigma$ model which links with the giant elliptical models by TBH, where a vehement collapse combined with an overwhelming single early starburst event occurs because any starformation self-regulation is disabled by the dominating gravitational energy density.

The radial distribution of star formation within the model is shown in Fig. 8. Prior (at an age of $400 \mathrm{Myr}$ ) to the initial giant starburst star formation is spread over almost the entire galaxy which has a radius of $\approx 10 \mathrm{kpc}$. During the initial burst and the plateau SFR phase the star forming region shrinks smoothly to about half the radius of the stellar galaxy. In the late starbursts, shown at 5 Gyr, young stars are strongly centrally concentrated, and the model would resemble a blue compact dwarf while the bursts were active. During the final decline star formation continues to be centrally concentrated because there is so little gas 


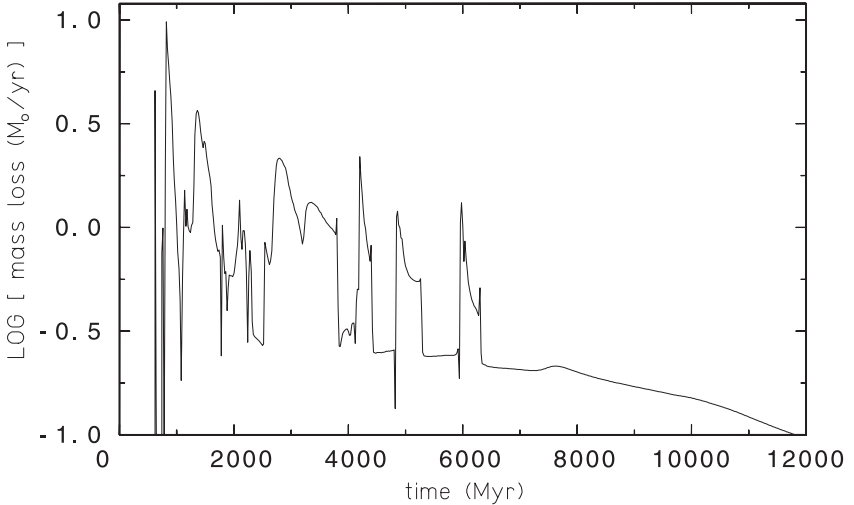

Fig. 9. Time-dependence of the rate of unbound mass with respect to the gravitational potential of the same $10^{10} M_{\odot}$ model as in Fig. 7. For discussion see text.

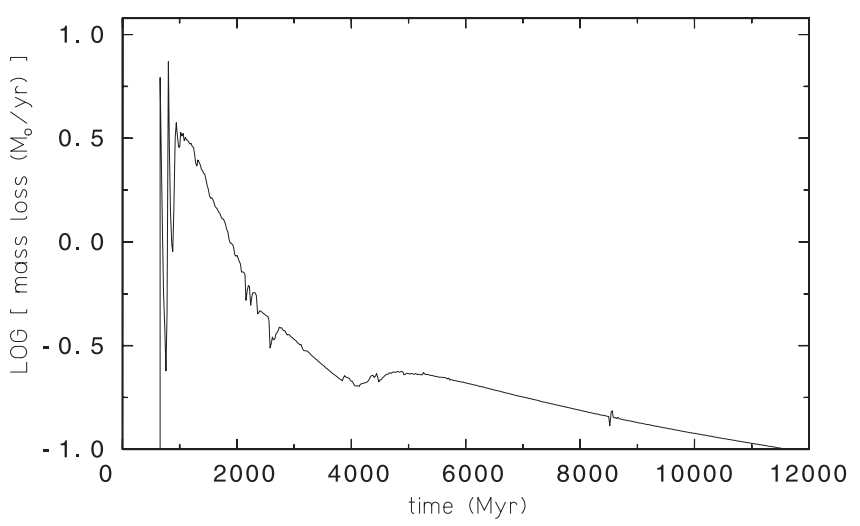

Fig. 10. Same as Fig. 9 but for a $10^{10} M_{\odot} 1 \sigma$ galaxy model with a $10 \%$ drag coefficient (see text).

that even a slow SN Ia explosion rate is sufficient to prevent dense CM gas build-ups in the system.

In Fig. 7 also the time-dependent behavior of the model ISM is shown. Note that the amount of hot ICM varies in accordance with the recent SFR and is kept hot at later times by SNe I. Mass loss continues throughout the lifetime of the model, as shown in Fig. 9. From this figure one also discerns that ICM mass and star formation from Fig. 7 show reasonably the same temporal behaviour as the mass-loss rate, but a time delay of the latter by almost 200-300 Myr is remarkable. The galactic mass-loss rate depends on specific features of the model, including the metallicity (which influences cooling times) and especially on the amount of drag force as hot gas flows through the CM. Figure 10 presents results from a model in which the drag force is increased to $10 \%$ as compared with the $1 \%$ coupling assumed for most of our models.

The radial density distribution of the long-living low-mass stars traces the dissipational evolution of the collapse. From Fig. 11 one recognizes that the radial brightness has not developed a single exponential profile. One can distiguish between three different regions in the simple log-linear diagram. The slope of $\log \rho(r) \propto a r$ behaves as follows: in the outermost region at radii $R \gtrsim 4 \mathrm{kpc} \log \rho(r)$ is flat with $a \approx-\frac{1}{3} \mathrm{kpc}^{-1}$; further inward the slope steepens to $a \approx-1 \mathrm{kpc}^{-1}$ and in the very center below $300 \mathrm{pc}$ almost to $-4 \mathrm{kpc}^{-1}$. Only the $\mathrm{CM}$ is

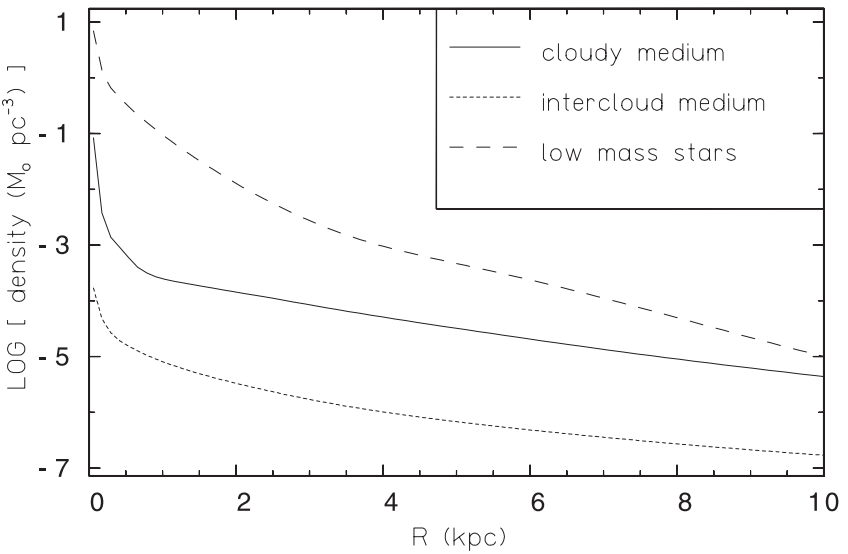

Fig. 11. Radial density distribution of cloudy and intercloud medium as well as low-mass stars after an age of $10 \mathrm{Gyr}$ of the same $10^{10} M_{\odot}$ model as in Fig. 7.

stratified almost exponentially until it steepens by more than 2 orders of magnitude within the innermost $500 \mathrm{pc}$, while the ICM follows more or less the square dependence of a freely expanding wind.

The drag force governs the degree of momentum coupling between hot ICM gas flows and the embedded but more massive cool gas clouds. In galaxy models of $10^{11} M_{\odot}$ this has a major impact on gas outflows (TBH). While in galaxies more massive than $10^{11} M_{\odot}$ the gravitational potential dominates the collapse independently of the drag coefficient, the level of gravitational binding of the ICM declines with decreasing mass of the galaxy model. Thus in lower mass galaxies drag coefficients in the reasonable range which we consider here have less effect on the amount of ICM mass loss due to galactic winds. The drag coefficient, however, does influence patterns of star formation in low-mass galaxies. Because higher drag coefficients introduce large-scale coherence within the galaxy, the $10 \%$ drag models do not suffer the centrally concentrated, later phase starbursts. The net effect of increased drag therefore is to smooth and slightly extend the evolutionary rate of the model (Fig. 11). In the end the final model contains almost $40-45 \%$ of the initial mass, and this is almost entirely in the form of stars.

We also calculate the time-dependent mean metallicity of the model components, which is calculated by including enrichment from SNeII and Ia. Figure 12 illustrates the radial metallicity for the components of the standard $10^{10} M_{\odot}$ model after 10 Gyr. While $Z$ of the low-mass stars as an observable varies radially between $\frac{1}{3}$ and $45 \%$ solar, the $C M$ lies around $\frac{1}{2} Z_{\odot}$ representing the observed interstellar medium metallicity.

\section{Observable consequences}

\subsection{Photometric evolution}

Due to the high initial SFRs associated with the early collapse of these models, all of the $\mathrm{dE}$ models are extremely luminous soon after formation. Assuming no obscuration due to dust, peak luminosities exceed $M_{B}=-23$ at about 0.3 to $0.5 \mathrm{Gyr}$ after the models begin to collapse. At this point the models are 


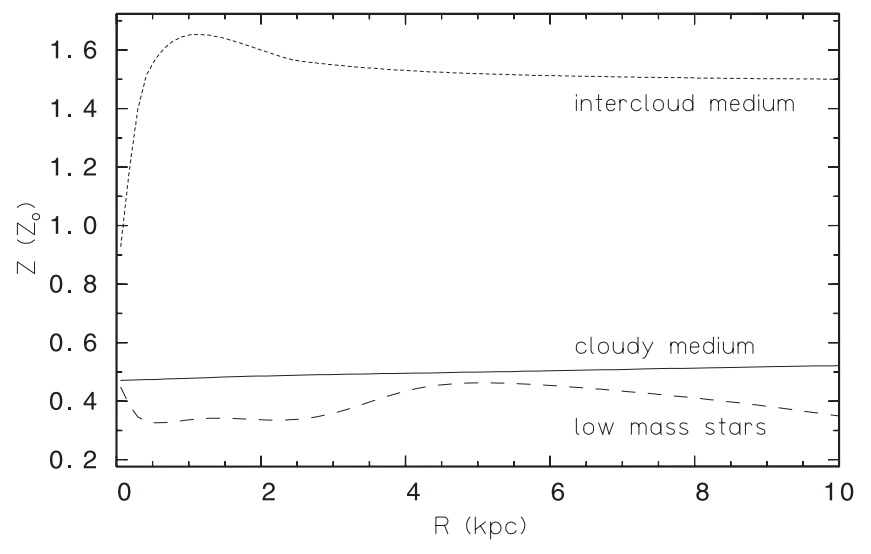

Fig. 12. Radial metallicity distribution of cloudy and intercloud medium as well as low-mass stars after an age of $10 \mathrm{Gyr}$ of the same $10^{10} M_{\odot}$ model as in Fig. 7.

also very blue (e.g., Loxen 1996), and therefore should be visible over cosmological distances.

Objects with this type of early evolution would not satisfy the requirements of Babul \& Ferguson (1996) for a highly visible phase of DGs at moderate redshifts. The best possibility for making these types of "faint blue galaxies" (Ellis 1997) would be from post-collapse SFR fluctuations (see Fig. 5). This type of model would be dominated by young stars near the peak of each burst, with the galaxy reaching $M_{B} \approx-19$ near the peak. These secondary starbursts should be blue and confined to the inner part of the model; they could yield faint blue galaxies with compact structures at redshifts near 1 .

A general feature of the $10^{10} M_{\odot}$ models is that star formation continues at significant levels nearly into the present epoch. Models like that shown in Fig. 4 will be relatively luminous and moderately blue. Using colors computed for us by J. Loxen (private communication), we predict $B-V$ colors of about 0.5 , bluer than are observed for most dEs. Models where star formation stops, such as that in Fig. 1, will be somewhat redder and can overlap the observed colors of $\mathrm{dE}$ galaxies. The less massive models cease star formation sooner and will therefore more closely match typical dEs in terms of colors and luminosities. However, we also note that this trend in the models would predict that more massive and therefore more luminous dEs should have bluer optical colors. This trend is not seen, although most of the photometric data come from cluster samples where external processes may have truncated star formation in all dEs (e.g., Secker et al. 1997). The issue of star formation in field $\mathrm{dE}$ galaxies is still to be fully explored.

\subsection{Metallicity}

Due to their rapid formation and efficient expulsion of gas on short timescales, our low mass models experience little stellar metal enrichment. For example, metal levels for most stars are predicted to be slightly below $10^{-2} Z_{\odot}$ in the $10^{9} M_{\odot}$ galaxy models. These have final stellar components with masses of $M_{*} \lesssim 10^{8} M_{\odot}$, corresponding to a current dE with $M_{V} \sim-15$. The $10^{10} M_{\odot}$ models (Fig. 7) have higher predicted stellar metallicities, about 0.3 of solar, and end with nearly 5-7 $\times$ $10^{9} M_{\odot}$.

Stellar metallicity distributions for Galactic dSph are largely derived from spectroscopic analyses of individual stars (see Gallagher \& Wyse 1994; Da Costa 1998; Mateo 1998, for reviews). The most reliable metallicities for other Local and $\mathrm{M} 81$ group $\mathrm{dSph} / \mathrm{dE}$ galaxies come from modeling of the red giant branch obtained with the Hubble Space Telescope (Caldwell et al. 1992, 1998; Han et al. 1997; Grebel et al. 2003), and from a few high-resolution spectra (e.g., Grebel \& Guhathakurta 1999; Shetrone et al. 2001; Tolstoy et al. 2003). While these interpretations are complicated by the presence of intermediate age stellar populations in many $\mathrm{dSph}$ and $\mathrm{dE}$ galaxies (Martínez-Delgado \& Aparicio 1998; Han et al. 1997; Mateo 1998; Grebel 1999), the results should be reasonably reliable, with the sense of any systematic errors being to overestimate the fractions of old, metal-poor stars (see Gallagher et al. 1998; Grebel et al. 2003).

A summary of recent stellar metallicity determinations for nearby dSph galaxies is presented by Grebel et al. (2003) who show that a strong correlation continues to exist between luminosity and mean stellar metallicity. This $\mathrm{L}-\mathrm{Z}$ correlation appears to be the hallmark of nearby spheroidal dwarfs. The metallicites of these systems range from $[\mathrm{Fe} / \mathrm{H}] \lesssim-2$ for low luminosity $\mathrm{dSph}$ galaxies with $M_{V} \gtrsim-10$ to $[\mathrm{Fe} / \mathrm{H}] \approx-0.5$ for the comparatively luminous $\mathrm{dE}$ companions of M 31 with $M_{V} \lesssim-15$. Most galaxies show a range in metal abundances, but the dispersions are modest, with $\sigma([\mathrm{Fe} / \mathrm{H}]) \approx 0.2-0.8$ (see e.g. Gallagher \& Wyse 1994; Han et al. 1997; Da Costa 1998; Caldwell et al. 1998, and references therein). Recent highresolution spectroscopy of red giants in several Galactic satellite dSphs (e.g., Shetrone et al. 2001; Tolstoy et al. 2003) shows the total range in metallicity of 1 dex and more, considerably exceeding the dispersion, and also indicates a wide range of stellar ages in several cases.

This comparison suggests that the low-mass $\mathrm{dE}$ models are slightly too metal-poor as compared with nearby $\mathrm{dSph} / \mathrm{dE}$ galaxy samples. Perhaps this could be a result of the locations of most of these galaxies near giant spirals? In the case of DM halos and/or external gas pressure by intergalactic gas the gas fraction lost by galactic winds would be diminished significantly. If mass loss, on the other hand, becomes less efficient the metal production can be extended and reaches higher values.

Secker et al. (1997) measured $B-R$ colors for a large sample of $\mathrm{dSphs}$ in the Coma cluster of galaxies. They find a strong correlation between color and luminosity that is completely consistent with the color-magnitude correlations derived for the Virgo and Fornax galaxy clusters (Caldwell \& Bothun 1987; Cellone et al. 1994), and with the $\mathrm{L}-\mathrm{Z}$ relationship derived from nearby dwarfs. While recent studies indicate that considerable scatter exists around this mean relationship in some clusters of galaxies (e.g., Rakos et al. 2001; Conselice et al. 2003a), the mean L-Z correlation is a fundamental characteristic of spheroidal dwarfs that all models must seek to match. In Fig. 13 the evolution of $Z_{\mathrm{CM}}$ with the temporally increasing stellar mass fraction is revealed for our five different mass and density models presented here. Since the 


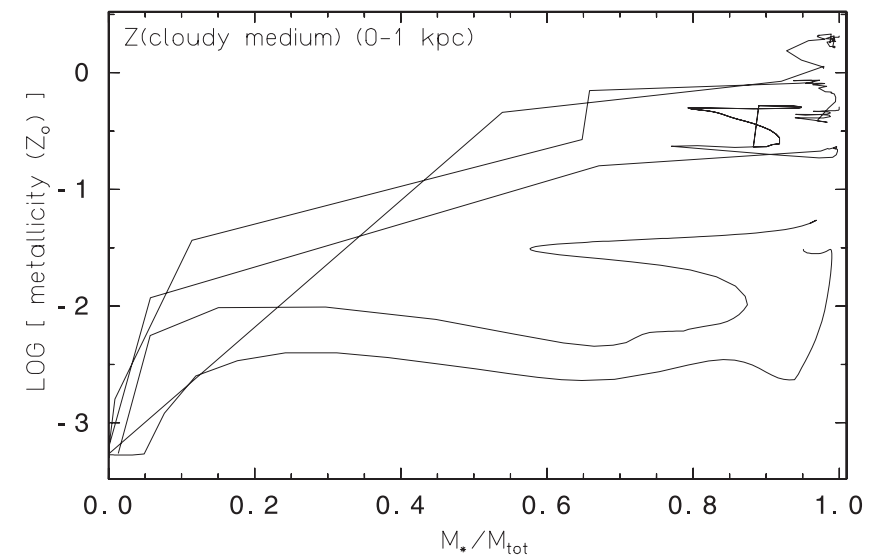

Fig. 13. Evolutionary tracks of the total metallicity $\mathrm{Z}$ of the cloudy medium within the inner $1 \mathrm{kpc}$ of the 5 models presented in this paper with increasing stellar mass fraction after $10 \mathrm{Gyr}$. From lowest to uppermost curve: $10^{9} M_{\odot}, 1 \sigma ; 10^{9} M_{\odot}, 3 \sigma ; 5 \times 10^{9} M_{\odot}, 1 \sigma ; 5 \times 10^{9} M_{\odot}$, $3 \sigma$; and $10^{10} M_{\odot}, 1 \sigma$.

$M_{*} / M_{\mathrm{tot}}$ ratio increases automatically if gas is expelled and lost, a horizontal track in this figure represents moreover the condition that no metal enrichment of the CM takes place. If star formation has ceased, mass loss of long-living stars with their original abundance composition even reduces $Z$, as visible in Fig. 13. Since stars that are formed from the CM and "are fossils containing a direct measure of abundances and their evolution since the earliest time" (Tolstoy \& Venn 2004), Fig. 13 is most appropriate to trace the evolution of dSphs and to derive conclusions about star-formation history and gas loss. For more massive dEs the chemo-dynamical models do show the proper trend of increasing metals with increasing stellar mass.

Two different external mechanisms that are not included in our models of isolated DGs affect their evolution towards the observed structures and should be distinguishable in the stellar metallicity: stripping of the ISM by motion through an intergalactic medium (Murakami \& Babul 1999; Grebel et al. 2003) would lead to metal loss, while gas confinement of the ISM by a static external pressure accelerates the evolution and enhances the metallicity (Vilchez 1995).

\subsection{Star formation histories}

\subsubsection{The initial starburst}

All of our models are dominated by an initial burst of star formation which follows within a few dynamical timescales of the initial collapse. We therefore predict that $\mathrm{dE}$ galaxies should flash into visibility as brilliant blue galaxies within a few hundred Myr of the beginning of their initial collapse. Taking the $1 \sigma$ fluctuation, $10^{10} M_{\odot}$ model as an example, the peak SFR averages about $60 M_{\odot} \mathrm{yr}^{-1}$ over about $30 \mathrm{Myr}$. This galaxy would have $M_{B} \approx-23$ near the end of the starburst, with blue optical and ultraviolet colors and strong emission lines. However, in the standard scenario this event would occur at a high redshift $z \geq 3$ and so would not contribute to the excess population of blue, star-forming galaxies seen at intermediate redshifts (e.g., Koo et al. 1995). However, these extreme bursts could be consistent with the properties of Lyman break galaxies which are seen at higher redshifts and may be associated with low mass objects (Somerville et al. 2001), although their redshifts are still too high if DGs undergo a prompt initial collapse $(z>8-10)$. Nevertheless, there is a chance for less massive DGs (e.g. $10^{9} M_{\odot}$ and less) where the formation of molecular hydrogen can be delayed to $z$ of almost 1.5 and later (Kepner et al. 1997) where they could also contribute to the populations of faint compact emission line galaxies.

Evidence for an initial starburst should also be detectable in the stellar populations of nearby $\mathrm{dE}$ systems. Unfortunately, the integrated spectra of $\mathrm{dE}$ galaxies have properties such that trade-offs between stellar ages and metallicities lead to uncertainties in both parameters (O'Connel 1996). However, the high precision color-magnitude diagrams for nearby dEs from the Hubble Space Telescope provide somewhat tighter constraints on star formation histories that will improve in quality as more direct measurements of stellar metallicities are obtained. These suggest, for example, in NGC 147 that star formation was extended over 5 Gyr or more, so that old, metal-poor as well as intermediate age and metallicity stellar populations are both present (Han et al. 1997). In NGC 147 the older stars are less centrally concentrated within the galaxy than the intermediate age stellar population. A similar spatial structure was found in the dSph And I by Da Costa et al. (1996), and subsequently was shown by Harbeck et al. (2001) to be a general feature of the Local Group population of dSph galaxies.

The currently available observations therefore indicate that $\mathrm{dE}$ galaxies have larger ranges in stellar ages than the simple models presented here. This difference could be understood if the initial starburst in the models is too rapid and violent. Reasons for this model behaviour are mainly due to the onedimensionality and the zero-angular momentum (see discussion below!). In addition, the initial chemo-dynamical model contains the same CM and ICM fractions in pressure equilibrium over the whole proto-galactic cloud. Since in reality these are encompassed by the intergalactic radiation field, the outskirts of the protogalaxy are exposed to ionizing radiation and therefore delayed in their collapse, while the central regime can cool and implode. This mitigates the collapse and therefore reduces the amplitude of the initial SFR peak. It is, therefore, unlikely that isolated dwarf galaxies undergoing a simple collapse could reach the SFRs seen in the Lyman break galaxies.

\subsubsection{Extended star formation}

Since the models do not eject all of their gas in the initial starburst, they are able to support an extended epoch of low level star formation. Even in one-dimensional models with momentum coupling between the outflowing hot ICM and cold clouds, the ISM is not totally removed during the early epochs of intense star formation. In a two- or three-dimensional model the evolution and by this mass loss could change. This issue can, however, not be predicted from simple linear estimates. The collapse of a rotating DG is smoothed down. By this, a weaker star formation and according SNII explosion rate would lead to a smaller amount of energy release and a less dramatic 
production of hot gas in overpressure. On the one hand, twodimensional structures like gaseous disks can collimate the hot gas outflow and, by this, enhance the galactic wind efficiency (MacLow \& Ferrara 1999).

A second robust prediction of these models is that $\mathrm{dE}$ galaxies should contain sufficient gas to support an extended period of star formation which will produce an intermediate age stellar population component. On the other hand, as we discussed earlier, there is extensive evidence for intermediate age stellar populations in dE galaxies. All of the M $31 \mathrm{dEs}$ contain intermediate age AGB stars, while NGC 185 and NGC 205 also have young Pop I stars (Hodge 1971; Da Costa 1998). In all cases the younger stellar components are somewhat centrally concentrated. The phenomological model predictions of extended low-level star formation, preferrentially occurring near the centers of dE galaxies, are consistent with the observed behavior of Local Group dE galaxies. They may also fit with the behavior observed in the dSph/dIrr "transition" galaxies which have dSph-like characteristics but support ongoing star formation (Grebel et al. 2003).

Another facet of intermediate age stars is their possible relationship to faint blue galaxies. Are the SFRs in dEs at intermediate epochs sufficiently high to produce excess numbers of blue galaxies, as proposed by Babul \& Rees 1992? The models with smooth post-birth SFRs clearly would not be seen at intermediate redshifts as faint blue galaxies. As emphasized by Babul \& Ferguson 1996, intense starbursts are necessary to make a small galaxy observable at redshifts of $z>0.5$. However, the highest amplitude post-formation bursts of star formation in the low drag models have adequate SFRs for these objects to be cosmologically visible, with SFRs of $\sim 1 M_{\odot} \mathrm{yr}^{-1}$ (Figs. 7, 4 and 5). Furthermore, our models indicate that this star forming activity will be confined to the central 2-3 kpc of the galaxies, and thus the angular sizes of the model during a starburst will be small. These events occur 4-6 Gyr after the initial collapse and could potentially be seen at redshifts of $z<1$.

Figure 11 provides an example of the predicted radial profile of a $10^{10} M_{\odot} \mathrm{dE}$ galaxy model. It has a relatively flat density profile that will yield a half-light radius of about $1 \mathrm{kpc}$, and would have a "Holmberg radius" of about $5 \mathrm{kpc}$; this is a very centrally concentrated system, and a similar effect is also seen in the $10^{9} M_{\odot}$ models, where a central light concentration is surrounded by a very extended, diffuse stellar halo. This behavior could give rise to the bright centers, "nuclei", that are found in more luminous dEs (e.g., Ferguson \& Sandage 1989; Binggeli \& Cameron 1991; Ferguson \& Binggeli 1994).

\subsection{The interstellar medium}

For the $10^{9} M_{\odot}$ models, an HI "halo" remains even as star formation ceases at large ages. Integrated over the entire galaxy, the mass of cool gas is comparable to that in stars, but in the central parts of the galaxy models the stars dominate the mass. The large gas content of our models is a direct consequence of the inclusion of a multi-phase representation of the interstellar medium. Hot gas is easily lost from these models, but cool gas clouds remain bound. An increase in the drag term that determines the momentum coupling in the models between hot and cool ISM phases increases the gas loss, but the high drag values required to produce extensive depletion of the ISM are not yet physically justified (see also Spaans \& Norman 1997). In all our models the CM density remains widely distributed over the luminous stellar content and even exceeds the stellar density in the $10^{9} M_{\odot}$ model at radii larger than $2 \mathrm{kpc}$ (see Fig. 2). While the CM density decreases almost exponentially outwards, it peaks steeply within the innermost few hundred parsecs (see Figs. 11 and 2). Therefore, conclusions based on dE galaxy models where the ISM is rapidly and completely lost via efficient supernova-powered galactic winds (e.g., DS86; Vader 1986, 1987; MacLow \& Ferrara 1999) should be reexamined in the light of the impact of a more realistic multi-phase ISM within such systems.

It is immediately evident that the models are not as gaspoor as most real dEs. This phenomenon has also emerged in the models by Carraro et al. (2001). Our models predict CM masses of $\gtrsim 10^{7} M_{\odot}$, which can be compared with HI mass limits of $\lesssim 10^{7} M_{\odot}$ for dE galaxies in the Virgo cluster (Bothun et al. 1985) but conflict with those even lower than $5 \times 10^{5} M_{\odot}$ (Conselice et al. 2003b). However, it is possible that the amount of diffuse ISM in Virgo dEs has been reduced by interactions with the hot intra-cluster medium. Nevertheless, an observed HI mass of only $4 \times 10^{5} M_{\odot}$ is found in NGC 205 (Young \& Lo 1997), the most gas-rich of the M $31 \mathrm{dE}$ family. NGC 205 also has a comparable mass of molecular gas (Young \& Lo 1996), but even so the total cool ISM mass is unlikely to exceed $10^{6} M_{\odot}$. The existence of relatively massive dEs with no detectable cool ISM, such as NGC 147 (upper limit of $3 \times 10^{3} M_{\odot}$; Young \& Lo 1997), is inconsistent with the present models and requires explanation. More sensitive searches for $\mathrm{HI}$ in local dSphs have manifested the surprising lack of neutral gas (Young 1999, 2000). Even more puzzling is its missing also in Fornax (Young 1999) where the age of the youngest stars requires their association with cool gas not more than $10^{8} \mathrm{yr}$ ago. We discuss possible sources for this discrepancy below.

\section{Conclusions and discussion}

We have presented results for one-dimensional models of dwarf spheroidal galaxies that collapse from $1 \sigma$ and $3 \sigma$ cosmological perturbations consisting only of baryonic matter. These models therefore experience rapid compression of their initial gas content and undergo violent initial starbursts. As anticipated by earlier models, such as DS86, this leads to extensive gas loss and a re-expansion of the remaining stellar system. However, due to the inclusion of a multi-phase representation of the ISM in our models, gas ejection is not complete and the model galaxies can sustain star formation for extended time periods (>5 Gyr) following the initial collapse for some choices of parameters. The initial behaviour is the same in the presence of a DM halo, while the dominance of the DM potential will capture the expanding gas.

These highly idealized models of $\mathrm{dE}$ galaxies show good qualitative fits to several key observations. They predict that $\mathrm{dE}$ galaxies will contain a range of stellar ages and stellar 
metallicities, and that dEs do not have to be gas-free systems, points that are consistent with some observations. Our models also qualitatively fit the observations in having increasing stellar metallicities at higher masses.

To make further progress it is important to explore the impact of the many approximations used for these models and to focus on those features which seem most at variance with the observations. These include:

1. The L-Z relationship for the models is not correct. The stellar metallicities predicted by our $10^{9} M_{\odot}$ models are about a factor of ten too low.

2. The models predict more cool gas than is observed in $\mathrm{dE}$ galaxies.

3. The models have cores which are very compact and implicitly high central surface brightnesses.

4. While the models make some intermediate age stars, most stars are made within a few free-fall times, i.e. in less than 1 Gyr after formation.

Many of these difficulties are likely to be associated with the very simple nature of these chemo-dynamical models:

1. As already mentioned above, the models are onedimensional. We would expect this to lead to overly efficient gas removal since we have forced the gas outflow to be isotropic.

2. Another argument also already cited above is the assumed rapid galaxy collapse without an extended period of inflow and no surrounding medium which could exert a pressure on the galaxy. These approximations are likely to be a major source of shortcomings in the models. Several lines of argument suggest that dwarf galaxies are likely to experience delays in gas infall due to the effects of ionization of the intergalactic medium, and that feedback from star formation within a dwarf can then further modulate late gas infall (Kepner et al. 1997; Spaans \& Norman 1997). Reducing the rate of infall would lower the peak star formation rate and has the potential to yield wider ranges of ages and metallicities by increasing the timescale for gas loss. They also may allow the initial epoch of major star formation to extend to lower redshifts, thereby increasing the fractions of intermediate age stellar populations and linking $\mathrm{dE}$ galaxy formation to the faint blue galaxy populations, as suggested by Babul \& Rees (1992). The downside of these modifications could be to increase the residual cool ISM content, which is already a problem even with our current violent mass loss models.

3. Our models represent isolated unperturbed galaxies where the inflated but still gravitationally bound gas is considered as part of the galaxy. This falsifies the mass fraction and leads to misinterpretations if compared with observations. Two effects will act jointly: at first, the removed gas will not be visible in the neutral state due to ionizing intergalactic radiation and heat conduction in a bath of hot intergalactic gas; secondly, gaseous halos can be easily torn out of the galactic gravitational potential wells of dwarfs by means of stripping when moving with respect to a surrounding intergalactic (Murakami \& Babul 1999; Ikuta 2001; Grebel et al. 2003).
4. The models do not contain dark halos. While the presence of dark halos in $\mathrm{dE}$ galaxies in the mass range that we have considered is uncertain, their presence will have an effect on gas loss. For example, Fukunaga-Nakamura $\&$ Tosa (1989) considered the effects of a dark halo on a $\mathrm{dE}$ model galaxy with extensive mass loss. They noted that some reduction in central baryonic mass concentration is likely even when the dark halo dominates.

5. There is no external radiation field in the model. As we noted in item 2, radiation can influence gas flows in low density regions of the galaxy. It will also modify the state of the predicted low density HI gas "halos" in our models, and could considerably reduce the amount of cool gas remaining in the models over a cosmic timescale. Ionized halo gas will remain undetectable until $\mathrm{H} \alpha$ surveys have become sensitive enough.

While more complex models are required to bring the qualitative agreement with observations into the quantitative regime, we are encouraged that an extension of this here presented chemo-dynamical approach can yield useful quantitative insights into the evolution of spheroidal dwarf galaxies. Beside the simplicity of this first approach to DGs the chemodynamical treatment provides a deeper insight into regulation and interaction processes of the stars with the multi-phase ISM and leads to deviations from simpler models that are understandable in the light of the description.

Acknowledgements. The authors gratefully acknowledge discussions with Joachim Köppen and Rosemary Wyse. The paper has largely benefitted from comments and discussions with the referee Nobuo Arimoto for a better clarification of the model issues. J.S.G.'s work on dwarf elliptical galaxies has been supported in part by the WFPC2 Investigation Definition Team under NASA contract NAS7-1260 to JPL and by the National Science Foundation through grant NSF AST 9803018.

\section{References}

Baade W. 1944, ApJ, 100, 137

Babul, A., \& Ferguson, H. C. 1996, ApJ, 458, 100

Babul, A., \& Rees, M. J. 1992, MNRAS, 255, 346

Barkana, R., \& Loeb, A. 1999, ApJ, 523, 54

Bender, R., \& Nieto, J.-L. 1990, A\&A, 239, 97

Bender, R., Paquet, A., \& Nieto, J.-L. 1991, A\&A, 246, 349

Berczik, P., Hensler, G., Theis, C., \& Spurzem, R. 2003, in Evolution of Galaxies. III. From simple Approaches to self-consistent Models, ed. G. Hensler et al., Ap\&SS, 284, 865

Binggeli, B., Sandage, A., \& Tarenghi, M. 1984, AJ, 89, 64

Binggeli, B., Sandage, A., \& Tammann, G. 1985, AJ, 90, 1681

Binggeli, B., \& Cameron, L. M. 1991, A\&A, 252, 27

Bothun, G. D., Mould, J., Wirth, A., \& Caldwell, N. 1985, AJ, 90, 697

Burkert, A., \& Hensler, G. 1989, Proc. Summer School on Evolutionary Phenomena in Galaxies, Tenerife, ed. J. E. Beckman \& B. E. J. Pagel (Cambridge University Press), 230

Caldwell, N. 1987, AJ, 94, 1116

Caldwell, N., \& Bothun, G. D. 1987, AJ, 98, 1542

Caldwell, N., Armandrof, T. E., Seitzer, P., \& Da Costa, G. S. 1992, AJ, 103, 840

Caldwell, N., Armandroff, T. E., Da Costa, G. S., \& Seitzer, P. 1998, AJ, 115, 535 
Carraro, G., Chiosi, C., Girardi, L., \& Lia, C. 2001, MNRAS, 327, 69

Cellone, S. A., Forte, J. C., \& Geisler, D. 1994, ApJS, 93, 397

Cellone, S. A., \& Forte, J. C. 1996, ApJ, 461, 176

Colless, M., Ellis, R. S., Taylor, K., \& Hook, R. N. 1990, MNRAS, 244, 408

Conselice, C. J., Gallagher, J. S. III., \& Wyse, R. F. G. 2001, ApJ, 559, 791

Conselice, C. J., Gallagher, J. S. III., \& Wyse, R. F. G. 2003a, AJ, 125, 66

Conselice, C. J., Gallagher, J. S. III., \& Wyse, R. F. G. 2003b, ApJ, 591,167

Da Costa, G. S., Armandroff, T. E., Caldwell, N., \& Seitzer, P. 1996, AJ, 112, 2576

Da Costa G. S. 1998, in Stellar astrophysics for the local group, VIII Canary Islands Winter School of Astrophysics, ed. A. Aparicio, A. Herrero, F. Sanchez, 351

Dekel, A., \& Silk, J. 1986, ApJ, 303, 39

Dufour, R. J., \& Hester, J. J. 1990, ApJ, 350, 149

Ellis, R. S. 1997, ARA\&A, 35, 389

Ferguson, H. C., \& Sandage, A. 1989, ApJ, 346, L53

Ferguson, H. C., \& Binggeli, B. 1994, A\&AR, 6, 67

Friaca, A. C. S., \& Terlevich, R. 1998, MNRAS, 298, 399

Fukunaga-Nakamura, A., \& Tosa, M. 1989, PASJ, 41, 953

Gallagher, J. S., III. 2003, in Evolution of Galaxies. III. From simple Approaches to self-consistent Models, ed. G. Hensler et al., Ap\&SS, 284, 1

Gallagher, J. S., \& Wyse, R. F. G. 1994, PASP, 106, 1225

Gallagher, J. S., Tolstoy, E., Dohm-Palmer, R. C., et al. 1998, AJ, 115, 1869

Grebel, E. K. 1997, Rev. Mod. Astron., 10, 29

Grebel, E. K. 1999, in The Stellar Content of the Local Group, ed. P. Whitelock \& R. Cannon, ASP Conf. Ser., IAU Symp., 192, 17

Grebel, E., \& Guhathkurta, P., 1999, ApJ, 511, 101

Grebel, E. K., Gallagher, J. S., \& Harbeck, D. E. 2003, AJ, 125, 1926

Guzman, R., Koo, D. C., Faber, S. M., et al. 1996, ApJ, 460, 5

Guzman, R., Jangren, A., Koo, D. C., et al. 1998, ApJ, 495, L13

Han, M., Hoessel, J. G., Gallagher, J. S., III., et al., 1997, AJ, 113, 1001

Harbeck, D., Grebel, E. K., Holtzman, J., et al. 2001, AJ, 122, 3092

Harfst, S., Theis, C., \& Hensler, G. 2003, in Evolution of Galaxies. III. From simple Approaches to self-consistent Models, ed. G. Hensler et al., Ap\&SS, 284, 869

Heckman, T. M., Armus, L., \& Miley, G. K. 1990, ApJS, 74, 833

Hensler, G. 1987, Mitt. Astron. Ges., 70, 141

Hensler, G. 2003, in CNO in the Universe, ed. C. Charbonnel et al., ASP Conf. Ser., in press

Hensler, G., \& Rieschick, A. 1999, in The Evolution of Galaxies on Cosmological Timescales, ed. J. E. Beckman \& T. J. Mahoney, ASP Conf. Ser., 187, 214

Hensler, G., Theis, C., \& Burkert, A. 1993, in Proc. 3rd DAEC Meeting The Feedback of Chemical Evolution on the Stellar Content in Galaxies, ed. D. Alloin \& G. Stasinska, 229

Hodge, P. W. 1959, PASP, 71, 28

Hodge, P. W. 1971, ARA\&A, 9, 35

Hodge, P. W. 1989, ARA\&A, 27, 139

Hurley-Keller, D., Mateo, M., \& Nemec, J. 1998, AJ, 115, 1840

Ikuta, C. 2001, Ph.D. Thesis, University of Tokyo

Ikuta, C., \& Arimoto, N. 2002, A\&A, 391, 55

Kepner, J. V., Babul, A., \& Spergel, D. N. 1997, ApJ, 487, 61
Köppen, J., Theis, Ch., \& Hensler, G. 1995, A\&A, 296, 99

Köppen, J., Theis, Ch., \& Hensler, G. 1998, A\&A, 328, 121

Koo, D. C., Guzman, R., Faber, S. M., et al. 1995, ApJ, 440, L49

Kormendy, J. 1985, ApJ, 295, 73

Larson, R. B. 1974, MNRAS, 169, 229

Larson, R. B., \& Tinsleyn B. M. 1978, ApJ, 219, 46

Lia, C., Carraro, G., \& Salucci, P. 2000, A\&A, 360, 76

Loxen J. 1996, Ph.D. Thesis, University of Göttingen

MacLow, M.-M., \& Ferrara, A. 1999, ApJ, 513, 142

Martínez-Delgado, D., \& Aparicio, A. 1997, ApJ, 480, L107

Martínez-Delgado, D., \& Aparicio, A. 1998, AJ, 115, 1462

Mateo, M. 1994, Proc. ESO/OHP Workshop Dwarf Galaxies, ed. G. Meylan \& P. Prugniel, ESO, Garching, 309

Mateo, M. 1998, ARA\&A, 36, 435

Meyer, D. M., Cardelli, J. A., \& Sofia, U. J. 1997, ApJ, 490, L103

Mori, M., Yoshii, Y., \& Nomoto, K. 1999, ApJ, 511, 585

Murakami, I., \& Babul, A. 1999, MNRAS, 309, 161

O'Connel, R. W. 1996, in Proc. of From Stars to Galaxies: The Impact of Stellar Physics on Galaxy Evolution, ed. C. Leitherer, U. Fritze-von-Alvensleben, J. Huchra, ASP Conf. Ser., 98, 3

Pagel, B. E. J. 1987, in The Galaxy, ed. G. Gilmore \& B. Carswell (Dordrecht: Reidel), 341

Pagel, B. E. J. 1997, Nucleosynthesis and Chemical Evolution of Galaxies (Cambridge University Press)

Pagel, B. E. J., Terlevich, R. J., \& Melnick, J. 1986, PASP, 98, 1005

Petersen, R. C., \& Caldwell, N. 1993, AJ, 105, 1411

Phillipps, S., Drinkwater, M. J., Gregg, M. D., \& Jones, J. B. 2001, ApJ, 560, 201

Rakos, K., Schombert, J., Maitzen, H. M., et al. 2001, AJ, 121, 1974

Samland, M., Hensler, G., \& Theis, Ch. 1997, ApJ, 476, 277 (SHT)

Secker, J., Harris, W. E., \& Plummer, J. D. 1997, PASP, 109, 1377

Shetrone, M. D., Cote, P., \& Sargent, W. L. W. 2001, ApJ, 548, 592

Smecker-Hane, T. A. 1997, in Proc. of Star Formation Near and Far, ed. S. S. Holt \& L. G. Mundy, 571

Smecker-Hane, T. A., Stetson, P. B., Hesser, J. E., et al. 1994, AJ, 108, 507

Somerville, R. J., Primack, J. R., \& Faber, S. M. 2001, MNRAS, 320, 504

Spaans M., \& Norman, C. A. 1997, ApJ, 483, 87

Stetson, P. B., Hesser, J. E., \& Smecker-Hane, T. A. 1998, PASP, 110, 533

Sung, E.-C., Han, C., Ryden, B. S., et al. 1998, ApJ, 499, 140

Tammann, G. A. 1982, in Supernovae: A Survey of Current Research, ed. M. J. Rees \& R. J. Stoneham (Dordrecht: Reidel), 371

Theis, Ch., Burkert, A., \& Hensler, G. 1992, A\&A, 265, 465 (TBH)

Thompsen, L. A., \& Gregory, S. A. 1993, AJ, 106, 2197

Thoul, A. E., \& Weinberg, D. H. 1996, ApJ, 465, 608

Tinsley, B. M. 1973, ApJ, 186, 35

Tolstoy, E., \& Venn, K. 2004, in Highlights in Astronomy Vol. 13, IAU 2003, ed. O. Engvold, in press

Tolstoy, E., Venn, K. A., Shetrone, M. D., et al. 2003, AJ, 125, 707

Vader, J. P. 1986, ApJ, 305, 669

Vader, J. P. 1987, ApJ, 217, 128

Vilchez, J. M. 1995, AJ, 110, 1090

Wirth, A., \& Gallagher, J. S., III. 1984, ApJ, 282, 85

Young, L. M. 1999, AJ, 117, 1758

Young, L. M. 2000, AJ, 119, 188

Young, L. M., \& Lo K. Y. 1996, ApJ, 462, 203

Young, L. M., \& Lo K. Y. 1997, ApJ, 476, 127 